\title{
Citrate Effects on Amorphous Calcium Carbonate (ACC) Structure, Stability, and Crystallization
}

\author{
Dominique J. Tobler,* Juan Diego Rodriguez-Blanco, Knud Dideriksen, Nicolas Bovet, \\ Karina K. Sand, and Susan L. S. Stipp
}

Understanding the role of citrate in the crystallization kinetics of amorphous calcium carbonate (ACC) is essential to explain the formation mechanisms, stabilities, surface properties, and morphologies of $\mathrm{CaCO}_{3}$ biominerals. It also contributes to deeper insight into fluid-mineral interactions, both in nature and for industrial processes. In this study, ACC formation and its crystallization are monitored in real time as a function of citrate (CIT) concentration in solution. Additionally, synchrotron radiation pair distribution function analyses combined with solid-state, spectroscopic, and microscopic techniques are used to determine the effect of CIT on ACC structure, composition, and size. Results show an increase in ACC lifetime coupled with an increase in CIT uptake by ACC and slight changes in ACC atomic structure with an increase in CIT concentration. ACC does not form at concentrations $\geq 75 \% \mathrm{CIT} / \mathrm{Ca}$ and vaterite is absent in all cases where $\mathrm{CIT}$ is present. These findings can be explained by $\mathrm{CIT}$ binding with $\mathrm{Ca}$ ions, thereby forming $\mathrm{Ca}-$ $\mathrm{CIT}$ complexes in solution and decreasing ACC and calcite saturation levels. The formation of CIT-bearing ACC with calcitic structure and the absence of vaterite formation suggest that these solution complexes form a calcite-type atomic arrangement while CIT probably also acts as a growth inhibitor.

understanding of how additives modify $\mathrm{CaCO}_{3}$ growth kinetics and the mechanisms that control precipitation is therefore a topic of considerable interest. ${ }^{[1-3]}$

The initial steps of $\mathrm{CaCO}_{3}$ crystallization can occur via the formation of a poorly ordered amorphous calcium carbonate (ACC) phase. Synthetically formed ACC is often short lived and quickly transforms to more stable crystalline $\mathrm{CaCO}_{3}$ polymorphs such as vaterite, calcite, and aragonite. ${ }^{[4,5]}$ In contrast, biogenic ACC can be stable for much longer, even over the entire lifetime of an organism. ${ }^{[6,7]}$ The enhanced stabilization of ACC has been explained by the incorporation of considerable amounts of magnesium, phosphate, and silicate, as well as the occlusion of associated proteins and other macromolecules. ${ }^{[8,9]}$ For example, ACC aggregates in the intestinal tract of the seabream, Sparus aurata, are stabilized by the incorporation of up to $54 \mathrm{~mol} \%$ Mg. ${ }^{[10]}$ Similarly, ACC formed in the exo-

\section{Introduction}

The formation of calcium carbonate $\left(\mathrm{CaCO}_{3}\right)$ minerals in natural environments frequently occurs in the presence of inorganic and organic compounds. These are known to strongly modify crystal structure, morphology, and properties, often leading to more resilient and stronger crystals. Detailed

Dr. D. J. Tobler, Dr. J. D. Rodriguez-Blanco,

Dr. K. Dideriksen, Dr. N. Bovet, Prof. S. L. S. Stipp

Nano-Science Center

Department of Chemistry

University of Copenhagen

Copenhagen 2100, Denmark

E-mail: dominique.tobler@nano.ku.dk

Dr. K. K. Sand

Physical Sciences Division

Pacific Northwest National Laboratories

Richland, WA 99352, USA

Dr. K. K. Sand

Molecular Foundry

Lawrence Berkeley National Laboratory

Berkeley, CA 94720, USA

DOI: 10.1002/adfm.201500400 skeleton and gastroliths of the crayfish, P. clarkii, contain phosphoenolpyruvate and 3-phosphoglycerate (intermediates of the glycolytic pathway), which were shown to be responsible for ACC stabilization. ${ }^{[1]}$ While numerous laboratory studies have quantified the effects of inorganic additives on ACC formation, stability, and crystallization, ${ }^{[9,12,13]}$ the role of biomolecules is less well constrained, mainly because of the vast diversity of organic compounds, their variety of composition, chain length, and structure. Highly carboxylated species have been shown to extend ACC lifetime, ${ }^{[3,14-16]}$ whereas molecules with a lower number of carboxyl groups, such as single unit amino acids, do not exert much control on ACC stability. ${ }^{[3,17]}$ However, a mechanistic understanding of how these organic molecules affect ACC composition, structure, and lifetime is still lacking.

This study reports on investigations of the role of citrate (CIT) in ACC formation and crystallization. CIT is an intermediate in the tricarboxylic acid cycle and can form during glycolysis. It is used in various industrial processes, for example, as a flavoring additive in food, as a cleaning and chelating agent, and as a scale inhibitor in pipes, boreholes, and subsurface reservoirs. ${ }^{[18]}$ More recently, CIT has been shown to be an ideal coating agent to protect and stabilize metallic nanoparticles and to control their size. ${ }^{[19]}$ The effect of CIT on $\mathrm{CaCO}_{3}$ polymorph selection and crystal growth rates has been examined 
in previous work ${ }^{[20-22]}$ and CIT has been shown to favor calcite precipitation, while vaterite formation is inhibited through growth inhibition. ${ }^{21,22]}$ Similarly, aragonite growth is considerably inhibited by the presence of CIT. ${ }^{[21]}$ In addition to a reduced growth rate, CIT also leads to a reduction in $\mathrm{CaCO}_{3}$ crystal size. ${ }^{[23]}$

The impact of CIT on ACC formation and crystallization is less well constrained. Gebauer et al. ${ }^{[22]}$ observed that CIT binds weakly to $\mathrm{Ca}$ ions, thereby leading to more $\mathrm{Ca}$ being associated in clusters, i.e., reducing the number of free calcium ions, compared with the pure system. ${ }^{[22]}$ This led to a delay in ACC formation, while particle nucleation itself still occurred at similar supersaturation levels, i.e., at the same concentration of free calcium ions as in the pure system. They concluded that although CIT delays ACC nucleation, the nucleation process itself is not significantly suppressed. Similar to observations in previous studies, vaterite formation was not observed and calcite was the only polymorph to form, following the breakdown of ACC. They argued that this confirms the formation of ACC with short range atomic order that reflects calcite structure. Sato et al. ${ }^{[11]}$ found elevated CIT concentrations in ACC extracted from the exoskeleton and gastroliths of crustaceans, and they also showed that ACC synthesized in the presence of CIT was more stable than pure ACC. ${ }^{[11]}$ These studies illustrate that CIT can considerably affect ACC formation and stability but there is little quantitative data and we still lack a mechanistic understanding of the role of CIT in ACC crystallization.

We investigated the effects of citrate on ACC crystallization using time-resolved UV-vis spectrophotometry combined with synchrotron radiation pair distribution function (PDF) analyses, x-ray photoelectron spectroscopy (XPS), x-ray diffraction (XRD), thermogravimetric analysis (TGA), differential scanning calorimetry (DSC), and electron microscopy. In particular, we quantified changes in the ACC atomic scale structure, composition, and stability as a function of CIT concentration, to increase understanding of its role in the formation and stabilization of biogenic $\mathrm{CaCO}_{3}$.

\section{Results and Discussion}

\subsection{ACC Crystallization}

The effect of CIT on ACC formation and crystallization kinetics in solution was monitored via the time-dependent change in the solution absorbance using UV-vis spectrophotometry, while the nature and composition of the $\mathrm{CaCO}_{3}$ phases were verified by x-ray diffraction (XRD) through regular sampling. Figure 1A shows the results of the spectrophotometric analyses for CIT concentrations between 0 and $13 \times$ $10^{-3} \mathrm{M}$, which correspond to an initial CIT/Ca ratio of $0-100 \%$. Figure 1B shows two example spectrograms, for systems with and without ACC formation, explaining the crystallization stages. Although absorption can be difficult to relate directly to the particle size distribution, ${ }^{[24]}$ the trends observed give very clear indications on the processes that occur. At $0 \% \mathrm{CIT} /$ Ca (pure system), ACC formed instantly, as illustrated by an immediate increase in turbidity, i.e., absorbance, when the
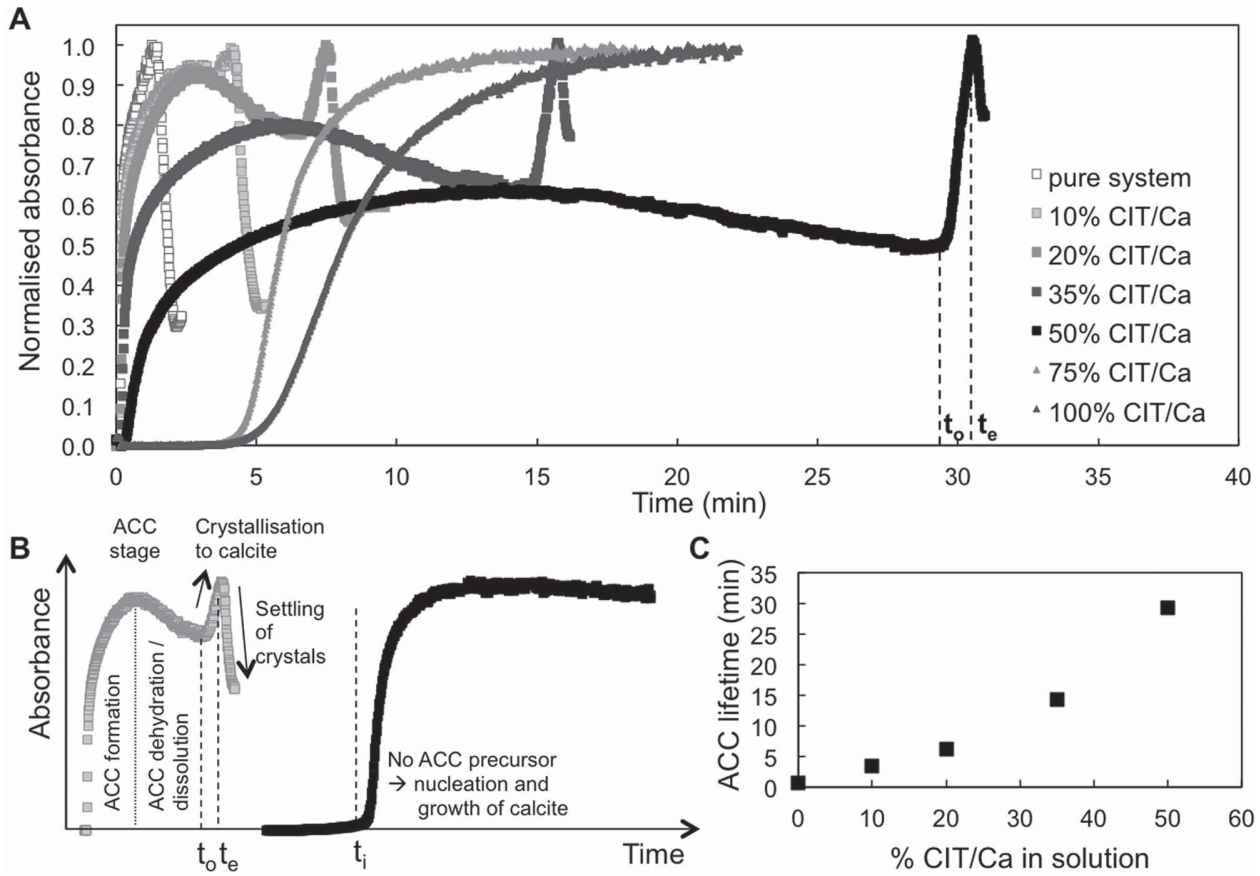

Figure 1. ACC formation and crystallization monitored by UV-vis spectrophotometry. A) Normalized absorbance ( $450 \mathrm{~nm})$ as a function of time after mixing two solutions of $13 \times 10^{-3} \mathrm{M} \mathrm{CaCl}_{2}$ and $13 \times 10^{-3} \mathrm{M} \mathrm{Na}_{2} \mathrm{CO}_{3}$, in a series of solutions with increasing proportions of CIT. B) The absorbance profile for $10 \%$ (grey squares) and $75 \%$ (black squares) $\mathrm{CIT} / \mathrm{Ca}$, with the various stages during crystallization explained; $t_{0}$ denotes the onset of calcite crystallization, $t_{\mathrm{e}}$ marks the end of crystallization and $t_{\mathrm{i}}$ is the induction time measured in systems, where calcite formed directly from solution, without an ACC precursor. These trends were verified by XRD analysis of samples removed at regular intervals. C) ACC lifetime, $t_{0}$, as a function of initial CIT/ Ca ratio in solution. 
Table 1. Data from spectrophotometric analyses and saturation indices predicted with PHREEQC, using concentrations from the solutions that were mixed. $t_{\mathrm{o}}$ and $t_{\mathrm{e}}$ mark the onset and completion of ACC transformation to calcite, and $t_{i}$ is the induction time measured in systems, where calcite formed directly from solution.

\begin{tabular}{|c|c|c|c|c|c|c|c|}
\hline \multirow{2}{*}{$\begin{array}{l}\mathrm{CIT} / \mathrm{Ca} \\
{[\%]}\end{array}$} & \multicolumn{3}{|c|}{ Time [min] } & \multicolumn{2}{|c|}{ Absorbance $(450 \mathrm{~nm})$} & \multicolumn{2}{|c|}{ Saturation index } \\
\hline & $t_{\mathrm{i}}$ & $t_{0}$ & $t_{\mathrm{e}}$ & at $t_{0}$ & at $t_{\mathrm{e}}$ & ACC & Calcite \\
\hline 0 & 0 & 0.6 & 1.3 & 1.82 & 2.1 & 0.75 & 2.82 \\
\hline 10 & 0 & 3.5 & 4.1 & 1.78 & 1.9 & 0.72 & 2.79 \\
\hline 20 & 0 & 6.3 & 7.5 & 1.31 & 1.7 & 0.69 & 2.75 \\
\hline 35 & 0 & 14.3 & 15.7 & 1.05 & 1.7 & 0.62 & 2.69 \\
\hline 50 & 0.17 & 29.2 & 30.5 & 0.71 & 1.5 & 0.54 & 2.61 \\
\hline 75 & 3.0 & - & - & - & 1.9 & 0.35 & 2.41 \\
\hline 100 & 3.4 & - & - & - & 2.0 & 0.11 & 2.17 \\
\hline
\end{tabular}

calcium and the carbonate solutions were mixed, at $t=0 \mathrm{~min}$. This ACC phase quickly crystallized to vaterite after about $50 \mathrm{~s}$, and eventually to calcite after 1-4 h (data not shown). With the addition of $10 \%, 20 \%$, and $35 \%$ CIT/Ca, ACC precipitation likewise began immediately when the solutions were mixed but ACC lifetime progressively increased with increasing CIT in solution (Figure 1A,C). At 50\% CIT/Ca, ACC formation was delayed by a short induction period of about $10 \mathrm{~s}$, where the mixed solutions remained clear, i.e., no absorbance detected. ACC that had formed under these high CIT concentrations also had the longest lifetime, i.e., it was almost two orders of magnitude more stable than the pure ACC (Figure 1A). All ACC formed in the presence of CIT transformed directly to calcite, with no vaterite intermediate, consistent with previous observations. ${ }^{[22,23]}$ At higher CIT concentrations, i.e., $75 \%$ and $100 \%$ CIT/Ca, there was no evidence of ACC formation. Instead XRD revealed the direct formation of calcite after an induction period of 3.0 for $75 \%$ and $3.4 \mathrm{~min}$ for $100 \%$ CIT/Ca. Comparison of the curves for these samples in Figure 1A shows a faster increase in absorbance (i.e., precipitation) for the sample with $75 \% \mathrm{CIT} / \mathrm{Ca}$, which means that calcite nucleated and grew faster in the $75 \% \mathrm{CIT} / \mathrm{Ca}$ solution than with $100 \% \mathrm{CIT} / \mathrm{Ca}$.

The increase in ACC lifetime $\left(t_{0}\right)$ with increasing CIT concentration in solution was mirrored by a gradual decrease in solution absorbance (i.e., turbidity) measured at $t_{0}$, where ACC transformation began (Table 1). For example, at $50 \% \mathrm{CIT} / \mathrm{Ca}$, the absorbance at $t_{\mathrm{o}}$ was 0.71 , which is less than half the value measured in the pure system (1.82). This shows that the volume of precipitated ACC decreased with increasing CIT/Ca. This can be explained by reduced activity of $\mathrm{Ca}^{2+}$, hence lower supersaturation with respect to pure ACC, resulting from $\mathrm{Ca}-\mathrm{CIT}$ complexing. Indeed, previous studies have shown that citrate acts as a chelating agent, binding with $\mathrm{Ca}$ ions. ${ }^{[22]}$ Such an effect is consistent with predictions from PHREEQC modeling, where we see a gradual decrease in saturation index for both ACC and calcite as CIT increases (Table 1). The PHREEQC modeling suggests that at
$75 \%$ and $100 \%$ CIT/Ca ACC is still supersaturated (Table 1) but the lack of ACC formation in these experiments could be a consequence of the supersaturation not being sufficient to overcome the nucleation barrier. Also, these calculations were made for the precipitation of pure ACC but if CIT is incorporated into ACC, its solubility product, and thus the calculated saturation index, would be different.

Similar to the decrease in the amount of precipitated ACC, a slight decrease in absorbance at $t_{\mathrm{e}}$, where ACC transformation to calcite is complete (Figure 1B), was observed with increasing CIT in solution (Table 1). For example, the absorbance at $t_{\mathrm{e}}$ was 1.4 times lower in the $50 \%$ CIT/Ca system than in the pure system. This trend matched the decrease in calcite saturation index as CIT increases, that is predicted with PHREEQC modeling (Table 1).

Another trend we observed in systems where CIT is present, is that during the ACC stage, the absorbance first increases to a maximum, then it decreases until crystallization begins at $t_{\mathrm{o}}$ (Figure 1A,B). This decrease in absorbance means that at some time following nucleation and growth of ACC, the volume of ACC in solution decreases, which is best explained by a decrease in particle size and/or number. One possibility is that aggregation and settling decrease the proportion of suspended particles, but this is unlikely because the solutions are continuously stirred. Indeed, even under static conditions, ACC particles remain in suspension for minutes to hours, depending on physicochemical conditions such as $\mathrm{T}, \mathrm{pH}$, and salinity. Another possibility is that ACC starts to dissolve and/or dehydrate prior the onset of crystalline $\mathrm{CaCO}_{3}$ formation. This has been suggested for the pure system in previous studies ${ }^{[5,25]}$ but has not yet been documented at such extended time scales.

\subsection{ACC Particle Size}

Transmission electron microscopy (TEM) analyses of ACC precipitates from all experiments showed that ACC particles consisted of fairly monodispersed spheres. Average size varied somewhat, depending on the proportion of CIT in the solution (Figure 2). In the pure system, ACC particles were $\approx 300 \mathrm{~nm}$ in diameter. The average particle size did not change with the addition of $10 \%$ CIT/Ca, while for $20 \%$ and $35 \%$
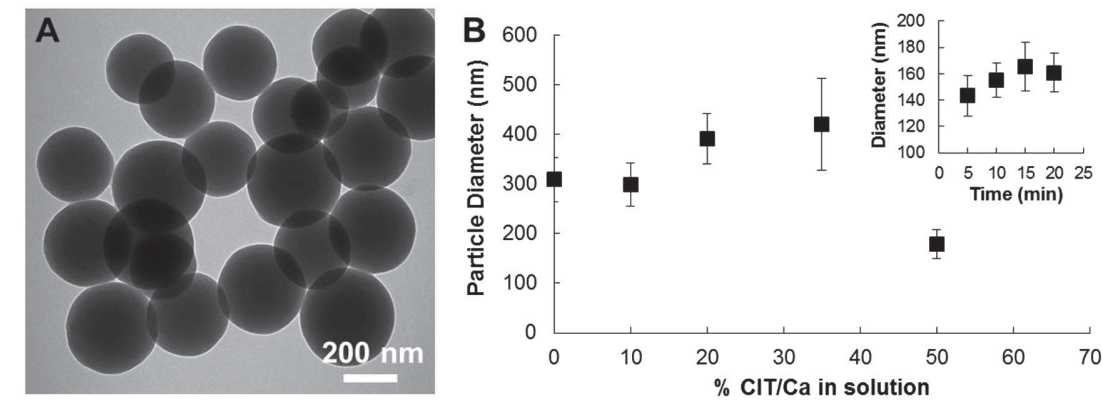

Figure 2. A) TEM image of ACC particles formed in the $10 \%$ CIT/Ca system. B) Average ACC particle diameter, determined from 100 to 150 particles in TEM images as a function of CIT/Ca ratio (error bars represent standard deviation). The inset shows ACC diameter as a function of lifetime in solutions with $50 \% \mathrm{CIT} / \mathrm{Ca}$. 
$\mathrm{CIT} / \mathrm{Ca}$, they were about $\approx 100 \mathrm{~nm}$ larger in diameter. When the citrate concentration was higher, i.e., at $50 \% \mathrm{CIT} / \mathrm{Ca}$, particles were smaller by $\approx 120 \mathrm{~nm}$ compared to the pure system (Figure 2B). Larger particle size can be explained by lower supersaturation with respect to ACC when the CIT/Ca concentration is higher, which would increase the magnitude of the nucleation energy barrier, thereby leading to fewer nucleation events, which would promote larger particles. ${ }^{[26]}$ However, the considerably smaller particle sizes observed in solutions with $50 \%$ CIT/Ca was unexpected. To verify that this particle size difference for the $50 \% \mathrm{CIT} / \mathrm{Ca}$ system is not a result of an experimental artifact, such as solution/solid separation at growth stages (i.e., different times) during ACC formation, we conducted a batch reaction with $50 \%$ CIT/Ca, where ACC samples were analyzed after 5, 10, 15, and 20 min (Figure 2, inset). Again, we observed average particle diameters $<200$ $\mathrm{nm}$ for all the samples, verifying that in solutions with $50 \%$ CIT/Ca, ACC particles are substantially smaller than in the other tested systems, independent of the ACC growth phase. This could be explained by the short induction period (10 s) and slower growth rate observed at $50 \% \mathrm{CIT} / \mathrm{Ca}$, which suggests that ACC nucleation and growth is inhibited considerably more than in solutions with lower CIT, thereby leading to slower growth rates. However, we are lacking a robust explanation for this at this point.

The time series in Figure 2B (inset) for the 50\% CIT/Ca system shows a similar trend as observed in the UV-vis spectrophotometry data (Figure 1A): the average ACC particle diameter increases during the first $15 \mathrm{~min}$ and then decreases slightly during the following $5 \mathrm{~min}$. It should be noted that the differences in diameter are not significant so it cannot be excluded that these changes may be the result of experimental artifact. However, it could also indicate that ACC particles decrease in size after an initial growth phase, which would support our earlier hypothesis that ACC dissolves or dehydrates before crystallizing to calcite. Time resolved $\mathrm{x}$-ray or light scattering could possibly provide higher resolution of the ACC particle size evolution.

\subsection{ACC Composition}

TGA and DSC analyses of pure ACC revealed an average weight loss of $19.3 \pm 0.3 \mathrm{wt} \%$ between 30 and $160{ }^{\circ} \mathrm{C}$, corresponding to continuous ACC dehydration until $\approx 160{ }^{\circ} \mathrm{C}$, where it transformed to calcite. This is seen in the exothermic peak in the DSC profile (Figure 3). The TGA profiles for the pure system showed a distinct two step weight loss: from 30 to $100{ }^{\circ} \mathrm{C}$ and from 130 to $160{ }^{\circ} \mathrm{C}$ (Figure 3). Similar TGA trends were observed in previous studies that have followed ACC dehydration as a function of increasing temperature. ${ }^{[3,12]}$ For example, Schmidt et al. ${ }^{[12]}$ observed a weight loss of $22.4 \mathrm{wt} \%$ for ACC synthesized by mixing equal volumes of $20 \times 10^{-3} \mathrm{M}$ calcium and $20 \times 10^{-3} \mathrm{M}$ carbonate solutions, with the two-step weight loss being attributed to a first fast release of weakly bound (mobile) water and a second step where structural water is released. They measured a crystallization temperature $\left(T_{\mathrm{c}}\right)$ of $\approx 200{ }^{\circ} \mathrm{C},{ }^{[12]}$ which is a little higher than what we observed. Such small differences in $T_{\mathrm{c}}$ can be explained by

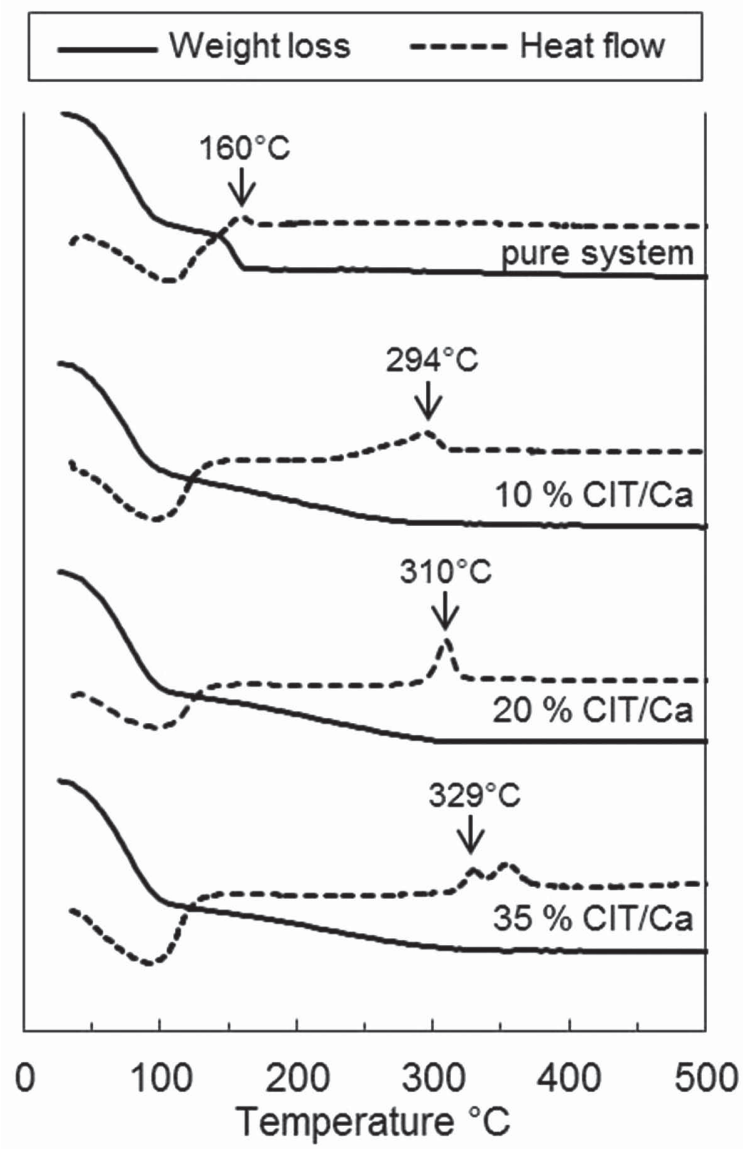

Figure 3. TGA and DSC analyses of ACC particles formed in the presence of $0 \%, 10 \%, 20 \%$, and $35 \%$ CIT/Ca. TCA profiles (solid lines) show gradual weight loss as a function of increasing temperature. DSC profiles (dashed lines) show heat flow during heating of the ACC samples, with arrows marking the temperature, where transformation to calcite occurred, i.e., the crystallization temperature $\left(T_{c}\right)$.

differences in ACC synthesis methods, instrument setup, and TGA running procedures.

TGA of ACC that formed in solutions with $10 \%, 20 \%$, and $35 \%$ CIT/Ca showed that the total weight loss did not differ much as a function of added CIT and was within error of the value obtained for the pure system (Table 2). The TGA profiles

Table 2. Data from TCA, elemental, and XPS analyses.

\begin{tabular}{|c|c|c|c|c|c|}
\hline \multirow{2}{*}{$\begin{array}{l}\mathrm{CIT} / \mathrm{Ca} \\
{[\%]}\end{array}$} & \multirow{2}{*}{$\begin{array}{l}\text { TGA weight } \\
\text { loss }{ }^{\text {a) }}[\mathrm{wt} \%]\end{array}$} & \multicolumn{2}{|c|}{ Elemental analyses ${ }^{\mathrm{b})}[\mathrm{wt} \%]$} & \multicolumn{2}{|c|}{ XPS atomic $\%$ ratioc) } \\
\hline & & C & $\mathrm{H}$ & $\mathrm{O}: \mathrm{C}_{2}$ & $C_{1}: C_{2}$ \\
\hline 0 & $19.3 \pm 0.3$ & 10.0 & 0.7 & 0.44 & 0 \\
\hline 10 & $19.6 \pm 0.4$ & 10.2 & 1.9 & 0.45 & 0.37 \\
\hline 20 & $20.1 \pm 0.8$ & 10.5 & 1.7 & 1.16 & 0.54 \\
\hline 35 & $19.8 \pm 0.6$ & 10.8 & 2.0 & 1.2 & 0.90 \\
\hline 50 & - & 11.2 & 1.5 & - & - \\
\hline
\end{tabular}

a) Determined from the $\%$ weight loss upon heating from 30 to $350{ }^{\circ} \mathrm{C}$; b) Standard deviation for total $\mathrm{C}$ and $\mathrm{H}$ analyses were 0.2 and $0.1 \mathrm{wt} \%$; ${ }^{\mathrm{c})} \mathrm{C}_{1}$ refers to $\mathrm{C}$ in the carboxylic group (XPS binding energy at $288.6 \mathrm{eV}$ ), whereas $\mathrm{C}_{2}$ represents $\mathrm{C}$ in $\mathrm{CO}_{3}$ (binding energy $290.1 \mathrm{eV}$ ). 
(Figure 3) however, were different than in the pure system. The initial rapid decrease of mobile water until $\approx 100{ }^{\circ} \mathrm{C}$ was identical but with further heating, mass was lost gradually up to temperatures between 290 and $330{ }^{\circ} \mathrm{C}$, with the crystallization temperature, $T_{\mathrm{c}}$, increasing as CIT increased (arrows, Figure 3). In contrast, in the pure system, the second major weight loss was considerably faster and ACC transformation to the crystalline polymorphs was complete at $160{ }^{\circ} \mathrm{C}$. This also means that between 100 and $160{ }^{\circ} \mathrm{C}$, pure ACC samples lost all structural water $(6.0 \mathrm{wt} \%)$, while ACC that had formed in the presence of CIT only lost 2.0-2.6 wt\% in this temperature range (Figure 3). This is consistent with CIT inhibiting the fast dehydration of the ACC particles, indicating that CIT is incorporated into the solid, possibly replacing water and even carbonate. As a result, a higher temperature is required to break down the dry CIT-bearing ACC. TGA analysis of citric acid monohydrate showed that it decomposes rapidly between 180 and $260{ }^{\circ} \mathrm{C}$ and loses about $80 \mathrm{wt} \%$, suggesting that some of the weight loss observed between 160 and $350{ }^{\circ} \mathrm{C}$ could stem from adsorbed and incorporated CIT. Moreover, the increase in crystallization temperature with increasing CIT/Ca (arrows, Figure 3) suggests that more CIT was incorporated as CIT concentration increased in the solution. This was further verified by visual inspection of the samples following TGA, which showed that the color of the remaining solids changed with proportion of CIT. Decomposed pure ACC was white, while the solids with CIT became darker gray with increasing CIT. TGA of the citric acid monohydrate alone resulted in a blackish residual, thus the darker color corresponds to more CIT associated with the solid.

Elemental ( $\mathrm{C}$ and $\mathrm{H}$ ) analyses offer an alternative way to determine changes in ACC composition as a function of varying CIT content. With this method, the total release of carbon (inorganic and organic) and hydrogen is measured as the sample is heated to $900{ }^{\circ} \mathrm{C}$. For pure ACC, a $10 \mathrm{wt} \% \mathrm{C}$ loss was observed (Table 2), which corresponds to inorganic $\mathrm{C}$ from carbonate. With the increase in CIT/Ca, an increase in $\mathrm{C}$ and $\mathrm{H}$ release (wt\%) was observed (Table 2), consistent with uptake of a compound that contains carbon and hydrogen, such as CIT.

We used XPS to probe the composition of the top $10 \mathrm{~nm}$ of the ACC particles. Figure 4 shows high-resolution XPS spectra for the electrons ejected from the $\mathrm{O} 1 \mathrm{~s}$ and $\mathrm{C} 1 \mathrm{~s}$ energy levels. Surface atomic ratios are presented in Table 2. The O 1s peak for pure ACC shows a distinct shoulder at higher binding energy (Figure 4A), which is expected for the presence of water. In the CIT-bearing samples, this shoulder increased with the CIT concentration in solution, which is also shown by the increase in the $\mathrm{O}: \mathrm{C}_{2}$ atomic ratio (Table 2). It is difficult to deconvolute the components from water, CIT, and carbonate in the oxygen peak. However, an increase in CIT was also identified in the C 1s peak (Figure 4B). Several subpeaks increased in intensity with an increase in CIT, including a peak with binding energy of $288.6 \mathrm{eV}$, which represents carbon in $\mathrm{O}-\mathrm{C}=\mathrm{O}$ bonds. The atomic ratio between $\mathrm{C}$ from the carboxylic group of citrate $\left(\mathrm{C}_{1}\right)$ and $\mathrm{C}$ from carbonate $\left(\mathrm{C}_{2}\right)$ increases with CIT, consistent with the TGA and the elemental analyses, in that the amount of sorbed, i.e., adsorbed and incorporated, citrate increased with increasing CIT/Ca in solution (Table 2).
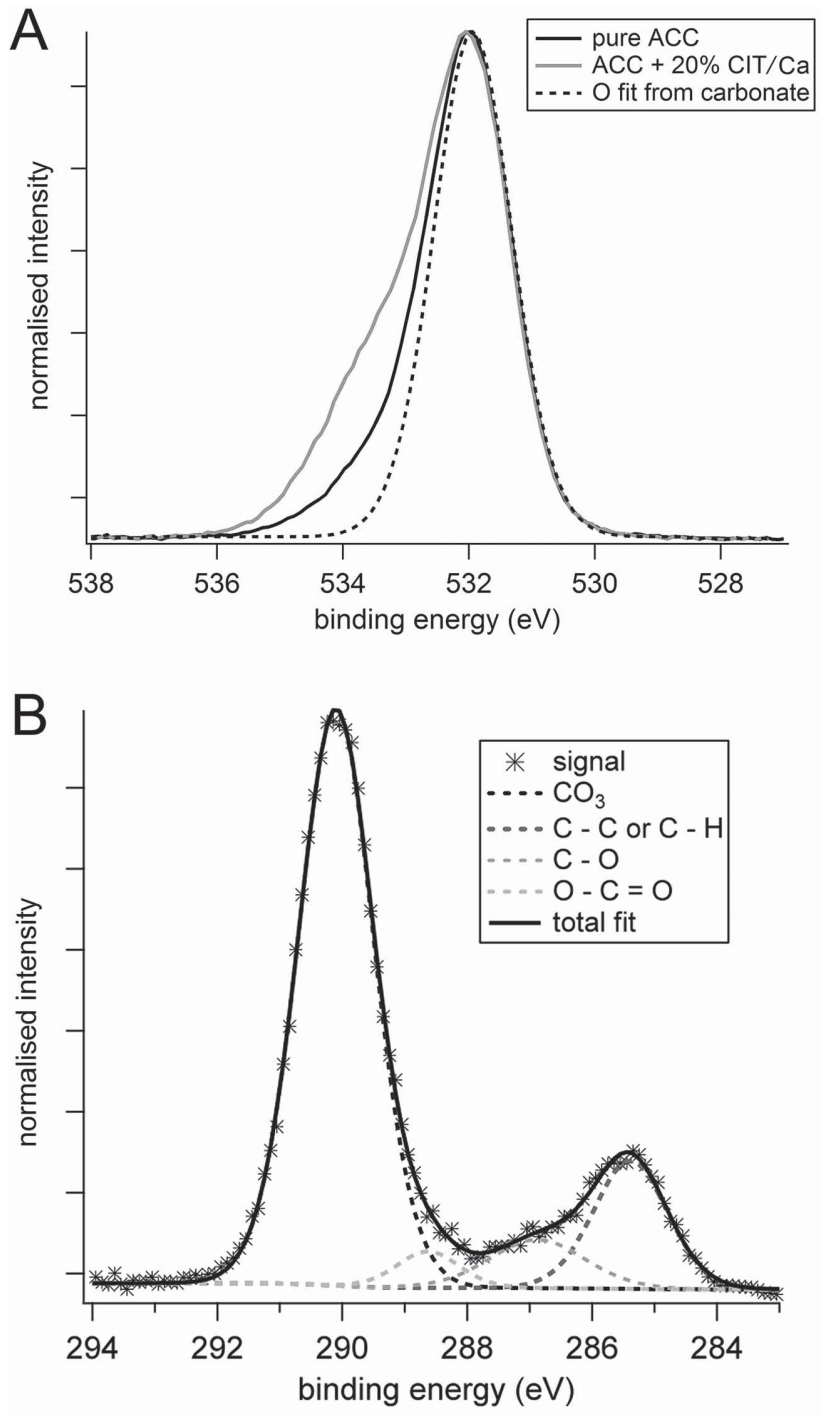

Figure 4. A) High-resolution XPS spectra for oxygen, $\mathrm{O} 1 \mathrm{~s}$, for pure ACC and ACC formed in the presence of $20 \% \mathrm{CIT} / \mathrm{Ca}$. B) High-resolution XPS spectra for carbon, $\mathrm{C}$ 1s, associated with $\mathrm{ACC}$ formed in solutions containing 20\% CIT/Ca. Fitted components are for the $\mathrm{C}-\mathrm{C}$ and $\mathrm{C}-\mathrm{H}$ bond (binding energy at $285.4 \mathrm{eV})$, the $\mathrm{C}-\mathrm{O}$ bond $(286.9 \mathrm{eV})$, the carboxylic group, $\mathrm{O}=\mathrm{C}-\mathrm{O}(288.6 \mathrm{eV})$ and $\mathrm{CO}_{3}(290.1 \mathrm{eV})$. The peaks at 286.9 and $285.4 \mathrm{eV}$ were observed on all samples (with/without CIT). These are common on all samples of calcium carbonate and are explained by adventitious carbon, i.e., contamination from the air, the solutions, and the rinse with isopropanol. Increase in the carboxylic peak at 288.6 however, is consistent with increased concentration of CIT.

\subsection{ACC Structure}

Synchrotron radiation PDF analysis of pure ACC and CIT-ACC showed very similar patterns, with well-defined peaks at $1.3 \AA$, the $\mathrm{C}-\mathrm{O}$ distance in carbonate, and at $2.4 \AA$, mostly from first neighbor $\mathrm{Ca}-\mathrm{O}$ atom pairs (Figure 5 ). There are also broader peaks at $\approx 4$ and $6 \AA$. Although these peaks have contributions from several atom pairs, they largely reflect electron denser Ca. ${ }^{[27]}$ The positions of the peaks match those reported in previous studies ${ }^{[12,27-29]}$ and confirm the amorphous character of the ACC synthesized in this study. This is particularly visible by 


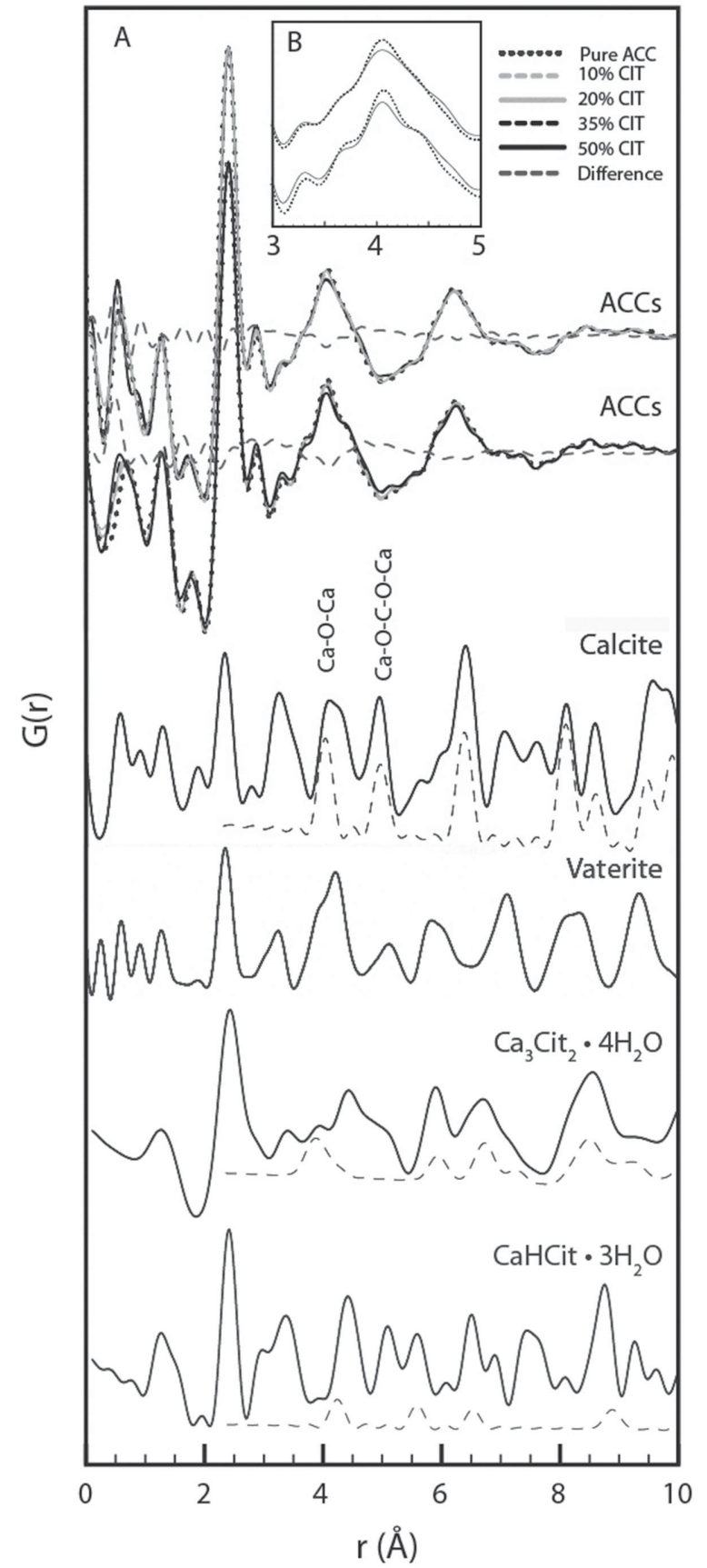

Figure 5. Measured PDFs of pure ACC and CIT-ACC, pure calcite and vaterite and calculated PDFs for $\mathrm{Ca}$-citrate salts. The two sets of PDF data for ACC were acquired during different beamtimes, to demonstrate reproducibility. For the ACC PDF sets, the dashed line represents the difference between the PDFs of the pure ACC and the $50 \% \mathrm{CIT}$ sample. For the $\mathrm{Ca}$-citrate solids, the PDFs of all atomic pairs and the contributions from $\mathrm{Ca}-\mathrm{Ca}$ atomic pairs (dashed line) were calculated from published structures. ${ }^{[30,31]}$ For calcite, the structure of Graf ${ }^{[32]}$ was first fitted to the experimental data before calculating the contributions from $\mathrm{Ca}-\mathrm{Ca}$ atomic pairs (dashed line). Because the vaterite structure is still being debated, calculated $\mathrm{Ca}-\mathrm{Ca}$ distances have not been added.

comparing with PDFs of the crystalline polymorphs calcite and vaterite, where atom pairs have well-defined positions at much higher separation, reflecting their crystalline nature. Although peak positions were not affected by CIT addition, i.e., no visible peak shifts in comparison with the pure sample, peak intensity varied slightly with CIT/Ca ratio (all profiles were normalized to have matching intensity for the $\mathrm{Ca}-\mathrm{O}$ peak at $2.4 \AA$ ). Specifically, a small decrease in peak intensity was observed at $r \approx 4$ and $6 \AA$, in particular at a CIT/Ca ratio of $50 \%$ (Fig. 5B). This peak intensity decrease was accompanied by an increase in intensity at about 3.2 and $5.2 \AA$. In other words, the peaks at 4 and $6 \AA$ are broader, presumably reflecting a diversification of the local bonding environment beyond the first shell when CIT ions are incorporated. This is consistent with data from XPS, TGA/DSC and elemental analyses. The changes in signal intensity are best illustrated by subtracting the pure ACC spectrum from the PDF spectrum acquired for the $50 \% \mathrm{CIT} / \mathrm{Ca}$ ACC (dashed line in Figure 5). These differences match poorly with the PDFs for crystalline Ca-citrates calculated from published data, ${ }^{[30,31]}$ indicating that the observed variations with CIT are not caused by the presence of such phases in the ACC. Moreover, varying the molecular formula of ACC (with water and CIT) that was used for PDF data normalization could not explain the variation in peak intensity and broadening.

Recently, it has been proposed that there is structural variation in different types of ACC. ${ }^{[22,25,28,33]}$ Gebauer et al. ${ }^{[22]}$ observed that the presence of CIT resulted in only calcite forming during ACC transformation and they proposed that CIT leads to clusters that have calcite structure. In our experiments, transformation to calcite is also clearly favored, indicating that CIT might be able to template calcite formation. To do so, the positions of the carboxylate oxygens in $\mathrm{Ca}$-CIT would need to mimic the pattern of the carbonate ions in calcite. Calcite structure requires octahedral coordination of $\mathrm{Ca}$, with the first neighbor $\mathrm{Ca}$ atoms separated by $\approx 4 \AA$. These Ca octahedra corners share an $\mathrm{O}$ atom in one carbonate and are bridged by another carbonate. The PDFs of both ACC and CIT-ACC feature a broad peak with maximum of $\approx 4 \AA$ (Figure 5), suggesting that calcite-like ordering, involving first neighbor $\mathrm{Ca}$ atoms, could easily be present in ACC. The second neighbor Ca atoms in calcite are separated by $\approx 5 \AA$. These octahedra are bridged by two carbonate groups of alternating orientation. In the PDF of the pure ACC, $r \approx 5 \AA$ marks the low point between the two broad peaks, suggesting that a calcite-like motif, involving second neighbor $\mathrm{Ca}$ atoms, are rare. On the other hand, the PDF of CIT-ACC shows a slight intensity increase at this value (Figure 5), which could represent the presence of some calcitelike motifs involving second neighbor $\mathrm{Ca}$ atoms. However, the presence of such motifs is not obvious.

If CIT acts as a template for generating specific interatomic distances that make the ACC more susceptible to transformation to calcite, such an effect would most likely also operate during calcite nucleation if it occurs homogeneously in solution or heterogeneously at the ACC surface. In a recent study to probe ACC formation and transformation in water with in situ TEM, calcite was never observed to form within ACC or at its surface. ${ }^{[34]}$ It is more likely that ACC transformation occurs via dissolution-reprecipitation. Our spectrophotometric data (Section 2.1) also support this mechanism. The ACC structure could therefore have less of an impact on the forming crystalline phase. From these results only however, we cannot deduce 
if this change in local structure governs the polymorph selection or if the impact of CIT on structures in solution, through formation of complexes and/or through decreased concentration of free calcium ions (i.e., decreasing the supersaturation with respect to calcite), drives calcite nucleation. In addition, the preferred ACC transformation to calcite in systems with added CIT could also be a result of the different dissolution kinetics of CIT-bearing ACC, which leads to different local saturation levels than for the pure system. Finally, previous studies ${ }^{[23]}$ indicate that CIT inhibits vaterite crystal growth considerably more than calcite growth because of more extensive growth site blocking on vaterite than on calcite. This can certainly contribute further to preventing vaterite nucleation and growth in the presence of CIT.

\subsection{Crystal Size and Morphology}

Scanning electron microscopy (SEM) images show that the presence of CIT has a profound effect on calcite crystal size and morphology (Figure 6). In the pure system, calcite crystals typically form imbricated twin clusters. Similarly, at $10 \%$ CIT, calcite crystals are rhombohedral and intertwined but the number of individual crystals within a cluster is higher and the crystals are smaller than in the pure system (Figure 6A,B). This indicates that calcite crystal growth is distinctly disturbed by the addition of CIT and suggests more nucleation sites on the calcite surface. This is even more evident at higher CIT concentration (20-50\%; Figure 6C,D). Crystal form is no longer

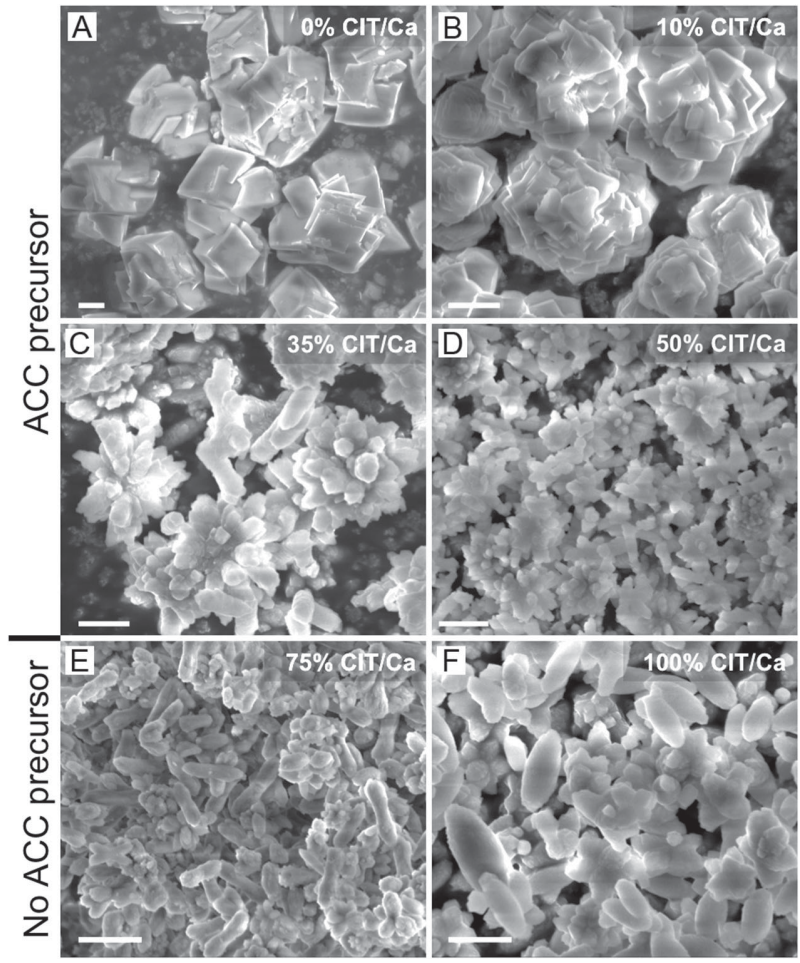

Figure 6. SEM images of calcite that had formed by $4 \mathrm{~h}$ in the various $\mathrm{CaCO}_{3}$ crystallization experiments with the $\mathrm{CIT} / \mathrm{Ca}$ ratio ranging from $0 \%$ to $100 \%$ : A) $0 \%$; B) $10 \%$, C) $35 \%$, D) $50 \%$, E) $75 \%$, and F) $100 \%$. The scale bar is $2 \mu \mathrm{m}$ for all images. rhombohedral. Nanocrystalline calcite subunits form elongated particles with rough surfaces. This morphology suggests spherulitic growth, ${ }^{[35,36]}$ where new crystals continuously nucleate on the existing crystals but with different orientation. Several other studies have described spherulitic growth, i.e., nucleation controlled growth, in carbonates formed after the breakdown of a poorly ordered precursor (e.g., vaterite, protodolomite). ${ }^{[2,5]}$ These morphologies have been explained by the large difference in solubility between the ACC and the crystalline polymorphs, i.e., as ACC dissolves, supersaturation for the crystalline $\mathrm{CaCO}_{3}$ polymorphs becomes high enough to promote nucleation at a constant rate.

Aside from the morphological changes, the size of the nanocrystalline subunits, as well as of the aggregates, i.e., spherulites, decreases as the proportion of CIT/Ca in solution increases. Thus as CIT/Ca increases, the number of calcite nuclei increases, producing many small crystals rather than fewer, larger ones, such as in the pure system. A possible explanation is that ACC precipitation is affected considerably more by the presence of CIT than calcite precipitation so once ACC transformation begins, the solutions with higher CIT/Ca ratio are more saturated with respect to calcite, leading to higher nucleation and crystallization rates.

At $75-100 \%$ CIT, where no ACC precursor formed and calcite precipitated from solution following a 3-3.5 min induction time, crystal form was similar to the calcite formed from ACC, in the other CIT experiments. Particles were elongated, composed of abundant nanocrystalline subunits, as is typical for spherulitic growth. However, particle dimension was generally a bit smaller in solutions of $75 \% \mathrm{CIT} / \mathrm{Ca}$ (Figure 6E) compared with 100\% CIT/Ca (Figure 6F). This is expected because calcite would nucleate at slightly lower supersaturation in the $100 \%$ solution (Table 1), which would lead to fewer nuclei and larger crystals.

\subsection{The Role of Citrate in ACC Formation and Crystallization}

The results demonstrate that citrate affects ACC local structure and has a significant impact on $\mathrm{CaCO}_{3}$ formation and crystallization. Citrate is well known to be a strong chelating agent for calcium ions, thus reducing the activity of free calcium ions in solution. In systems supersaturated with respect to ACC and calcite, the removal of free calcium ions into complexes decreases the level of supersaturation (Table 1), which in turn decreases the amount of precipitated ACC and calcite. Our spectrophotometric measurements combined with XRD analyses support this, showing a decrease in the amount of ACC formed with increasing CIT/Ca from $0 \%$ to $50 \%$ and ultimately the complete inhibition of ACC formation at CIT/Ca $\geq$ $75 \%$ (Figure 1). Although less ACC forms as CIT/Ca increases in solution, we observe a steady increase in ACC lifetime. We argue that this enhanced ACC stability results from CIT uptake by the ACC (XPS, TGA, and elemental analyses, Figures 3 and 4), which is further supported by the increase in ACC crystallization temperature with increasing CIT/Ca (DSC, Figure 3). Thus, we propose that CIT that is present on the crystal surfaces and within ACC particles retards dehydration and decomposition compared with the pure system. 
In the pure system, $\mathrm{ACC}$ transformation to crystalline $\mathrm{CaCO}_{3}$ is characterized by the simultaneous dissolution of ACC and precipitation of vaterite and calcite. In the presence of citrate, vaterite formation is inhibited and ACC transforms directly to calcite. It is important that as CIT/Ca increases in solution, the total amount of ACC formed steadily decreases, which means that the remaining total concentration of $\mathrm{Ca}$ and $\mathrm{CO}_{3}$ in solution increases, increasing the supersaturation level. Prior to the breakdown of the CIT-ACC, the saturation index for calcite is highest in solutions with $50 \%$ CIT/Ca. We conclude that these higher saturations levels are a consequence of the inhibition of calcite nucleation by the high citrate concentration in solution. Once crystallization begins, the higher calcite supersaturation levels in high CIT/Ca solutions lead to a faster ACC transformation, i.e., higher nucleation rate. This is supported by the morphology and dimension of the forming calcite crystals. We observe spherulitic calcite growth, where the diameter of the spherulites decreases with increasing CIT in solution.

At CIT/Ca ratio $\geq 75 \%$, no ACC formed. We observed direct nucleation and growth of calcite following an induction period. This suggests that at these high CIT concentrations, the CITACC ion activity product (which is a function of $\mathrm{Ca}, \mathrm{CO}_{3}$, and CIT concentrations) is lower than the CIT-ACC solubility product. However, the supersaturation with respect to calcite is highest under these conditions (direct precipitation from solution, i.e., not by way of ACC crystallization) and the high CIT concentration inhibits calcite formation, ${ }^{[21]}$ which explains the longer induction time than for the pure system. Once crystallization begins, growth occurs rapidly, driven by the high supersaturation, which leads to a nucleation dominated growth process that favors spherulitic calcite. Under these conditions, where no ACC precursor forms, the rate of calcite formation essentially depends on the solution CIT concentration. This means that the calcite nucleation barrier increases with increasing CIT concentrations, leading to the formation of larger spherulites with increasing CIT, i.e., fewer nuclei form and these can grow large before the solution reaches calcite equilibrium.

The results of this systematic quantification of the lifetime of ACC in the presence of CIT clearly demonstrate that citrate stabilizes ACC in solution and promotes its crystallization to calcite, consistent with previous reports. ${ }^{[11,22]}$ Citrate has so far only been associated with the formation and enhanced stability of biogenic ACC in crustaceans but because of its ubiquity, it might also play a role in ACC stabilization and transformation kinetics in other ACC producing plants and animals. Comparison with our previous work ${ }^{[17]}$ shows that citrate has a far greater impact on ACC formation, lifetime, and crystallization kinetics, in particular its thermal stability, than simple amino acids, such as aspartate, aminoacetate, and glutamate. On the other hand, the uptake, i.e., the adsorption and incorporation, of citrate in ACC have similar effects as if $\mathrm{Mg}$ ions were added to the system, both increasing ACC lifetime and thermal stability. $[2,3,9,37]$ The discovery that the presence of CIT promotes the formation of calcite crystals with different morphologies than in the pure system has not been described in detail before. It has implications for the interpretation of biomineral formation as well as for industrial processes, where calcite particles with specific dimension and morphology are required (e.g., paper, pigments, ceramics, drugs, filters, and abrasives).

\section{Experimental Section}

$\mathrm{CaCO}_{3}$ Synthesis and Crystallization: All ACC samples were synthesized at $25{ }^{\circ} \mathrm{C}$ by mixing (under constant stirring) equal volumes of a $13 \times$ $10^{-3} \mathrm{M} \mathrm{CaCl}_{2}$ solution and a $13 \times 10^{-3} \mathrm{M} \mathrm{Na}_{2} \mathrm{CO}_{3}$ solution with added $\mathrm{CIT}$ (ranging from 0 to $13 \times 10^{-3} \mathrm{M}$, i.e., $0-100 \% \mathrm{CIT} / \mathrm{Ca}$ ). Solutions were prepared using reagent grade chemicals and ultrapure deionized water (MilliQ, resistivity $>18 \mathrm{M} \Omega \mathrm{cm}$ ). Citrate-containing carbonate solutions were $\mathrm{pH}$ adjusted to $11.2(2 \mathrm{M} \mathrm{NaOH})$ to match the $\mathrm{pH}$ of the pure $\mathrm{Na}_{2} \mathrm{CO}_{3}$ solution

The effect of CIT on ACC formation and crystallization kinetics in solution was investigated by monitoring the time-dependent change in the solution absorbance using UV-vis spectrophotometry following the method described in previous work. ${ }^{[17,38]}$ This was done by mixing the $\mathrm{CaCl}_{2}$ and $\mathrm{Na}_{2} \mathrm{CO}_{3}$ (with added $\mathrm{CIT}$ ) solutions inside a plastic cuvette placed in the spectrophotometer (STS-UV, OceanOptics). During the measurement, the solution was continuously stirred and the absorbance (at $450 \mathrm{~nm}$ ) was monitored at $1 \mathrm{~s}$ intervals. In replica experiments, cuvettes were removed from the setup at regular time steps to determine the nature of the precipitate by using powder XRD. To collect the solid portion, the samples were filtered using vacuum filtration, the solid rinsed with isopropanol to remove remaining water and dissolved salts, and then quickly dried by blowing air over the solid. ${ }^{[39]}$ The dry samples were characterized with XRD and the results compared to the absorbance profiles, to link changes in absorbance to ACC lifetime and the onset and completion of crystallization.

Preparation of dry ACC samples for characterization using XPS, PDF, TCA, and DSC analyses was carried out by mixing larger solution volumes followed by rapid separation of solid and solution using vacuum filtration as described above. For systems with $0 \%, 10 \%$, and $20 \% \mathrm{CIT} / \mathrm{Ca}$, the solutions were filtered within $20 \mathrm{~s}$ of mixing, while at $35 \%$ and $50 \% \mathrm{CIT} / \mathrm{Ca}$, solutions were filtered within 2 min of mixing. It is unlikely that this time difference in sampling affected the solid ACC analyses because no differences in ACC structure and composition were observed for ACC samples removed at different time steps from the $50 \% \mathrm{CIT} / \mathrm{Ca}$ system (analyzed with PDF, TCA, and DSC). The dry ACC samples were first characterized using XRD to confirm the amorphous nature of the sample, then examined using XPS.

The saturation indices of ACC and calcite were calculated with the hydrogeochemical code PHREEQ ${ }^{[40]}$ using the minteq.v4 database ${ }^{[41,42]}$ and the solubility products of pure ACC ${ }^{[43]}$ and calcite. ${ }^{[44]}$ The saturation index, $\Omega$, is defined here as $\Omega=\mathrm{IAP} / \mathrm{Ksp}$, with IAP and Ksp being the ion activity product and thermodynamic solubility product.

$\mathrm{CaCO}_{3}$ Characterization: The local atomic structure of ACC that is formed in the presence of CIT was probed using synchrotron radiation PDF analyses. For comparison, PDFs were also acquired for calcite and vaterite synthesized in a pure system. PDF analyses were carried out at beamline 11-D-B (58.6 keV, $\lambda=0.2114 \AA$ ) at the Advanced Photon Source, Argonne National Laboratory, USA. The sample-detector geometry was calibrated with a $\mathrm{CeO}_{2}$ standard. Kapton capillaries were filled with ACC samples and measured for 5 min. An empty capillary was measured prior to the sample for background correction. Data were converted from 2D to 1D using the program Fit-2D. ${ }^{[45]}$ The experimental total scattering data were then processed using PDFget $X 2^{[46,47]}$ to get the $I(q)$ and PDF, or $G(r)$. This included standard corrections such as subtraction of background and contributions from incoherent scattering, normalization by the average atomic scattering cross section of the sample, and corrections to account for nonlinear detector efficiency. The molecular formula used for data normalization was $\mathrm{CaCO}_{3} \cdot \mathrm{H}_{2} \mathrm{O}$. The PDFs for the ACC samples were calculated from the Fourier transform of the reduced structure function truncated at $17 \AA^{-1}$. To probe $\mathrm{Ca}-\mathrm{Ca}$ distances in crystalline solids, theoretical PDFs were generated with the software PDFgui, ${ }^{[48]}$ a "real" space analogue to Rietveld refinement. The program allows calculations of PDFs from published structures as well as fitting of crystal structures to match theoretical PDFs with those measured. Theoretical PDFs of all atomic pairs and the contributions from $\mathrm{Ca}-\mathrm{Ca}$ atomic pairs were calculated using structures from the literature. For calcite, scaling, correlated atomic movement, unit cell 
dimensions, and isotropic displacement factors were first fitted to the experimental data.

The surface composition of ACC was quantified with cryo XPS, using a Kratos Axis Ultra ${ }^{D L D}$ system fitted with a monochromatic $\mathrm{Al}_{k} \alpha$ x-ray source $(h v=1486.6 \mathrm{eV}$, power $=150 \mathrm{~W})$. Base pressure in the ultrahigh vacuum (UHV) chamber was in the $10^{-9}$ mbar range. Cryogenic conditions kept the ACC in its pristine, hydrated state. Samples were rapidly vitrified in the vacuum chamber load lock by inserting the sample stub onto a precooled sample holder $\left(-170^{\circ} \mathrm{C}\right)$. This minimizes ACC transformation to crystalline phases during $\mathrm{x}$-ray irradiation and from loss of volatile molecules (i.e., water) during exposure to UHV. Pass energies of 10 and $160 \mathrm{eV}$ were used for high resolution and wide scans. Data were analyzed using the commercial software CasaXPS and its associated sensitivity factor library. Binding energies were calibrated by setting the $C$ is peak from carbonate to $290.1 \mathrm{eV} \cdot{ }^{[49]}$

The water and CIT content of ACC were determined using TGA (Netzsch TG 209 F1 Libra), where samples were heated at a rate of $10^{\circ} \mathrm{C} \mathrm{min}^{-1}$ from 30 to $800^{\circ} \mathrm{C}$ under $\mathrm{N}_{2}$ atmosphere. ACC samples were also analyzed using DSC (Netzsch DSC 214 Polyma) to determine the crystallization temperature for ACC transformation to calcite. For this, ACC samples were heated from 30 to $500^{\circ} \mathrm{C}$ at the same rate as for TCA measurements $\left(10^{\circ} \mathrm{C} \mathrm{min}^{-1}\right)$ and under $\mathrm{N}_{2}$ atmosphere.

$\mathrm{CaCO}_{3}$ polymorphs were identified by XRD (Bruker D8, Co $\mathrm{K} \alpha 1,2$ radiation, $0.02^{\circ} \mathrm{step}^{-1}$ from 10 to $70^{\circ} 2 \theta, 1^{\circ} \mathrm{min}^{-1}$ ) and imaged using SEM (FEI Quanta 3D) and TEM (Philips CM20). To obtain solid samples at various times during the crystallization reaction, the reacting aqueous suspensions were filtered, the solids were rinsed with isopropanol and dried prior to analyses (as described in Section 2.1). The dry samples were placed on an aluminum SEM stub with sticky carbon tape and imaged at high vacuum with $10 \mathrm{kV}$. The diameter of the ACC was determined with TEM. To prepare the samples, ACC was resuspended in isopropanol and then a drop was placed on a copper TEM grid, left to dry, and imaged at $200 \mathrm{kV}$. We used selected area electron diffraction (SAED) to verify the amorphous nature of ACC.

\section{Acknowledgements}

The authors thank Heloisa N. Bordallo and Marianne Lund Jensen for access to and support with TGA/DSC measurements, Birgitta Kegel for elemental analyses, and Henning Osholm Sørensen for help with writing the APS beamtime proposal. The authors are very grateful for the assistance provided by Karina Chapman, Peter Chupas, Rick Spence, and Kevin A. Beyer at APS beamline 11 ID-B. K.K.S. acknowledges the Danish Research Council for the Sapere Aude Fellowship. This work was supported by the Engineering and Physical Sciences Research Council [Grant No. EP/1001514/1] through the program grant for the Materials Interface with Biology (MIB) Consortium. The TCA/DSC analyses were enabled through a Carlsberg Foundation grant [No. 2013_01_0589]. Use of the Advanced Photon Source was supported by the U.S. Department of Energy, Office of Science, Office of Basic Energy Sciences, under Contract No. DE-AC02-06CH11357. Support for travel to the synchrotron facilities came from the Danish Council for Independent Research (via DANSCATT)

Received: January 30, 2015 Revised: March 7, 2015 Published online:

[1] F. C. Meldrum, H. Coelfen, Chem. Rev. 2008, 108, 4332.

[2] J. D. Rodriguez-Blanco, S. Shaw, P. Bots, T. Roncal-Herrero, L. G. Benning, Geochim. Cosmochim. Acta 2014, 127, 204.

[3] J. Ihli, Y.-Y. Kim, E. H. Noel, F. C. Meldrum, Adv. Funct. Mater. 2013, 23, 1575.

[4] J. D. Rodriguez-Blanco, S. Shaw, L. G. Benning, Nanoscale 2011, 3, 265.
[5] P. Bots, L. G. Benning, J.-D. Rodriguez-Blanco, T. Roncal-Herrero, S. Shaw, Cryst. Growth Des. 2012, 12, 3806.

[6] L. Addadi, S. Raz, S. Weiner, Adv. Mater. 2003, 15, 959.

[7] L. Gago-Duport, M. J. I. Briones, J. B. Rodriguez, B. Covelo, J. Struct. Biol. 2008, 162, 422.

[8] A. Akiva-Tal, S. Kababya, Y. S. Balazs, L. Glazer, A. Berman, A. Sagi, A. Schmidt, Proc. Natl. Aacd. Sci. USA 2011, 108, 14763.

[9] J. D. Rodriguez-Blanco, S. Shaw, P. Bots, T. Roncal-Herrero, L. G. Benning, J. Alloys Compd. 2012, 536, S477.

[10] E. Foran, S. Weiner, M. Fine, Sci. Rep. 2013, 3, 1700.

[11] A. Sato, S. Nagasaka, K. Furihata, S. Nagata, I. Arai, K. Saruwatari, T. Kogure, S. Sakuda, H. Nagasawa, Nat. Chem. Biol. 2011, 7, 197.

[12] M. P. Schmidt, A. J. Ilott, B. L. Phillips, R. J. Reeder, Cryst. Growth Des. 2014, 14, 938.

[13] A. V. Radha, T. Z. Forbes, C. E. Killian, P. U. P. A. Gilbert, A. Navrotsky, Proc. Natl. Acad. Sci. USA 2010, 107, 16438.

[14] X. R. Xu, A. H. Cai, R. Liu, H. H. Pan, R. K. Tang, K. W. Cho, J. Cryst. Growth 2008, 310, 3779

[15] B. Guillemet, M. Faatz, F. Grohn, G. Wegner, Y. Gnanou, Langmuir 2006, 22, 1875.

[16] L. B. Gower, Chem. Rev. 2008, 108, 4551.

[17] D. J. Tobler, J. D. R. Blanco, K. Dideriksen, K. K. Sand, N. Bovet L. G. Benning, S. L. S. Stipp, Proc. Earth Planet. Sci. 2014, 10, 143.

[18] H. Ashassi-Sorkhabi, E. Asghari, M. Mohammadi, J. Mater. Eng. Perform. 2014, 23, 2992.

[19] J.-W. Park, J. S. Shumaker-Parry, J. Am. Chem. Soc. 2014, 136, 1907.

[20] M. M. Reddy, A. R. Hoch, J. Colloid Interface Sci. 2001, 235, 365.

[21] K. Westin, A. C. Rasmuson, J. Colloid Interface Sci. 2005, 282, 359.

[22] D. Gebauer, H. Coelfen, A. Verch, M. Antonietti, Adv. Mater. 2009, $21,435$.

[23] K. J. Westin, A. C. Rasmuson, Desalination 2003, 159, 107.

[24] W. Haiss, N. T. K. Thanh, J. Aveyard, D. G. Fernig, Anal. Chem. 2007, 79, 4215.

[25] D. Gebauer, M. Kellermeier, J. D. Gale, L. Bergstrom, H. Coelfen, Chem. Soc. Rev. 2014, 43, 2348.

[26] W. Stumm, J. J. Morgan, Aquatic Chemistry, Chemical Equilibria and Rates in Natural Waters, Wiley, New York 1996.

[27] F. M. Michel, J. MacDonald, J. Feng, B. L. Phillips, L. Ehm, C. Tarabrella, J. B. Parise, R. J. Reeder, Chem. Mater. 2008, 20, 4720.

[28] A. V. Radha, A. Fernandez-Martinez, Y. D. Hu, Y. S. Jun, G. A. Waychunas, A. Navrotsky, Geochim. Cosmochim. Acta 2012, $90,83$.

[29] A. F. Wallace, L. O. Hedges, A. Fernandez-Martinez, P. Raiteri, J. D. Gale, G. A. Waychunas, S. Whitelam, J. F. Banfield, J. J. De Yoreo, Science 2013, 341, 885

[30] E. Herdtweck, T. Kornprobst, R. Sieber, L. Straver, J. Plank, Z. Anorg. Allg. Chem. 2011, 637, 655.

[31] B. Sheldrick, Acta Crystallogr. B 1974, 30, 2056.

[32] D. L. Graf, Am. Mineral. 1961, 46, 1283.

[33] D. Gebauer, P. N. Gunawidjaja, J. Y. P. Ko, Z. Bacsik, B. Aziz, L. Liu, Y. Hu, L. Bergstrom, C.-W. Tai, T.-K. Sham, M. Eden, N. Hedin, Angew. Chem. Int. Ed. 2010, 49, 8889.

[34] M. H. Nielsen, S. Aloni, J. J. De Yoreo, Science 2014, 345, 1158.

[35] J. P. Andreassen, J. Cryst. Growth 2005, 274, 256.

[36] L. Granasy, T. Pusztai, G. Tegze, J. A. Warren, J. F. Douglas, Phys. Rev. E 2005, 72, 011605.

[37] J. D. Rodriguez-Blanco, S. Shaw, L. G. Benning, Am. Mineral. 2015, DOI: 10.2138/am-2015-4693.

[38] J. D. Rodriguez-Blanco, B. Vallina, J. A. Blanco, L. G. Benning, Mineral. Mag. 2014, 78, 1373.

[39] J. D. Rodriguez-Blanco, S. Shaw, L. G. Benning, Mineral. Mag. 2008, 72, 283. 
[40] D. L. Parkhurst, US Geological Survey Water-Resources Investigations 1995, p. 143.

[41] V. Herndon, MINTEQA2/PRODEFA2, A Geochemical Assessment Model for Environmental Systems: User Manual Supplement for Version 4.0, HydroGeoLogic, Inc. \& Allison Geoscience Consultants, Inc., Athens, GA, USA 1998.

[42] J. D. Allison, D. S. Brown, K. J. Novo-Gradac, MINTEQA2/PRODEFA2, A Geochemical Assessment Model for Environmental Systems: Version 3.0 User's Manual, Environmental Research Laboratory, Office of Research and Development, U.S. Environmental Protection Agency, Athens, GA, USA 1991.

[43] L. Brecevic, A. E. Nielsen, J. Cryst. Growth 1989, 98, 504
[44] L. N. Plummer, E. Busenberg, Geochim. Cosmochim. Acta 1982, 46, 1011.

[45] A. P. Hammersley, S. O. Svensson, A. Thompson, Nucl. Instrum. Methods Phys. Res. A 1994, 346, 312.

[46] P. J. Chupas, X. Y. Qiu, J. C. Hanson, P. L. Lee, C. P. Grey, S. J. L. Billinge, J. Appl. Crystallogr. 2003, 36, 1342.

[47] X. Qiu, J. W. Thompson, S. J. L. Billinge, J. Appl. Crystallogr. 2004, 37,678 .

[48] C. L. Farrow, P. Juhas, J. W. Liu, D. Bryndin, E. S. Božin, J. Bloch, Th. Proffen, S. J. L. Billinge, J. Phys.: Condens. Matter 2007, 19, 335219.

[49] S. L. Stipp, M. F. Hochella, Geochim. Cosmochim. Acta 1991, 55, 1723. 